\title{
Enablers and barriers affecting medication-taking behaviour in aging men with benign prostatic hyperplasia
}

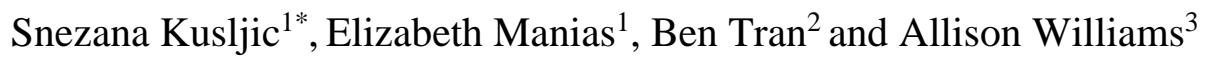 \\ ${ }^{1}$ Department of Nursing, The University of Melbourne, Australia; ${ }^{2}$ Department of Medicine, \\ The University of Melbourne, Australia; ${ }^{3}$ School of Nursing and Midwifery, Monash \\ University, Australia
}

Short Title: Medications and benign prostatic hyperplasia

$\underline{\text { Key words: }}$ adherence, benign prostatic hyperplasia, co-morbidities, language barrier, medication-taking.

* To whom correspondence should be sent at present address:

Dr Snezana Kusljic

Department of Nursing

The University of Melbourne

Parkville, VIC 3052, Melbourne, Australia

Tel: +61383449428

Fax: +61393474375

Email: skusljic@unimelb.edu.au 


\section{Abstract}

Objectives: To identify the enablers and barriers affecting medication-taking behaviour in aging men with benign prostatic hyperplasia.

Methods: A total of 40 patients attending the urology outpatient clinic in Melbourne in 2012 were screened. Patients who successfully met the inclusion criteria were interviewed using a structured interview schedule. Information regarding the patient's medication, demographic data and presence of co-morbidities was collected. Content analysis was compared with patient demographic and medical data, contributing to the analysis.

Results: Problems with medication-taking were reported in 58\% of patients. All patients without co-morbidities reported issues regarding their medications, whereas only $27 \%$ of patients with co-morbidities reported concerns regarding their medications. Statistical analysis revealed that patients without co-morbidities were significantly more likely $(\mathrm{p}=0.002)$ to have complaints with their medications compared to those with co-morbidities. Furthermore, patients with co-morbidities who required help of caregivers to assist with their medicationtaking were significantly less likely $(\mathrm{p}=0.05)$ to have complaints with their medications compared to patients who self managed.

Conclusions: Older patients with caregivers who assisted managing their medication-taking had better adherence. Those receiving aid from their caregivers were significantly less likely to have complaints regarding their medications as opposed to those not requiring a caregiver. This highlights the importance of having support for medication-taking in patients with comorbidities to assist with better adherence. 


\section{Introduction}

Benign prostatic hyperplasia or $\mathrm{BPH}$ is an age-dependant condition characterised by enlargement of the prostate gland and an increase in prostate contractility [1, 2]. These two pathological features cause urethral obstruction contributing to lower urinary tract symptomatology (LUTS) in aging men [3]. LUTS can range from voiding problems such as post-micturition dribble, frequent urination, and nocturia to incontinence, urinary tract infections and sexual impairment [4]. BPH affects around 50\% of men aged 50 years and by 85 years of age, $90 \%$ of men will have this condition [5].

Due to the increasing aging population, the prevalence of BPH in the community is expected to rise [6]. Coupled with rising healthcare costs, the effects of BPH and LUTS on men's quality of life can place an enormous burden onto society in terms of emotional, physical and economical suffering [7]. Since the early 90s, the development of pharmacological therapies has shifted the first-line therapy for BPH from surgery to medication [8]. There are a few common classes of medications used in the management of LUTS including $5 \alpha$-reductase inhibitors (5a-RIs), $\alpha$-blockers, anticholinergics and plant extracts, such as saw palmetto [9$11]$.

$5 \alpha$-RIs such as finasteride and dutasteride reduce the prostate size; however, their clinical efficacy is delayed by approximately 3-6 months [12]. $\alpha$-blockers such as prazosin and tamsulosin inhibit prostate contractility [13]. Clinical efficacy of $\alpha$-blockers is generally observed within 48 hours; however, these medications have no effect on prostate size and do not counteract further prostate growth [14]. Anticholinergic medications such as oxybutynin counter the bladder instability however many often experience moderate to severe side effects such as dry mouth, dizziness and constipation $[11,15,16]$. Plant extracts as a pharmacological therapy for the treatment of LUTS has a long tradition in some European countries [10] however their mechanism of action in the treatment of LUTS remains unclear. 
As medication has become the first-line therapy for the management of $\mathrm{BPH}$, reports have suggested that the adherence to some of these medications has been relatively poor. Unfortunately, reasons for this lack of adherence are not well documented [8, 17-19].

Adherence can be defined as the extent to which people follow the instructions they are given for prescribed treatments [20]. Yet, approximately 50\% people do not take their medications as prescribed with non-adherence increasing in the presence of chronic disease [20]. Consumers make deliberate rational and irrational decisions regarding their medications based on their beliefs about their illness and treatment. Rational decisions that contribute to the degree to which a consumer intentionally or unintentionally takes medications as prescribed are based on the concepts of purposeful action, patterned behaviour and feedback [21]. Purposeful action is the degree to which consumers decide to take their medications based on perceived need, effectiveness and safety. Patterned behaviour is the degree to which people initiate and establish a behavioural habit, routine or pattern of taking their medications through medication access, routine and remembering. Feedback is often used by consumers to evaluate the need for medications and is an incentive to take medications as prescribed.

Pound et al. (2005) synthesis of qualitative studies on medication adherence found that people were concerned about taking medications for long-term chronic diseases due to potential adverse effects [22]. As a result, individuals 'actively resisted' taking their medications, and concealed modifications they made to their medications, rather than forgetting to take them, as reported in other research [23]. Other reasons for lack of medication adherence include the cost of medications, disagreement about the need for treatment, and a poor partnership between consumers and healthcare professionals [20].

Most research into medication adherence has taken a biomedical approach, where interventions to enhance medication adherence encouraged education about the physiology of the chronic condition without consideration of the consumer's viewpoint [24]. Thus, the aim 
of the present study was to identify the enablers and barriers affecting medication-taking behaviour in aging men with BPH. 


\section{Methods}

The present study is the first of this kind to examine the enablers and barriers affecting medication-taking behaviour in aging men with $\mathrm{BPH}$. A total of 40 patients attending the urology outpatient clinic at a large metropolitan hospital in Melbourne, Australia were screened. Patients were selected through urology referrals who met the inclusion criteria. Only patients who were currently taking medication for BPH or symptoms associated with their prostate condition were invited to be interviewed. At recruitment, patients were given a verbal overview of what the study entailed and a detailed written plain language statement to carefully consider before agreeing to participate. The study was approved by the Human Resource Ethics Committee of Melbourne Health, Australia (Ethics approval QA2012034). Twenty-one patients were excluded from the study. The reasons for exclusion ranged from failure to identify prescribed medications, medications for prostate conditions were recently ceased by the prescriber, to being a new patient just commencing pharmacotherapy. Thus, the total number of patients to have successfully met the inclusion criteria was 19 . The structured interview took place after a routine appointment with the patients' urologist at the hospital. The structured interview focused on a set of 10 questions outlined in Appendix 1, and ranged from 20-35 minutes in length. The questions were aimed to gather information regarding the patients' behaviour towards medication-taking in relation to their livelihood and knowledge of their current condition and medications. The patients were given the opportunity to express any concerns they had regarding their current medications as part of the structured interview. The present study lends itself to a combined approach for qualitative and quantitative style of collection and statistical analyses. Comprehensive handwritten notes were taken during interviews to capture what patients said. Content analysis of patients' responses was undertaken. This analysis examined patients' words, phrases or sentences with similar

meanings which were then grouped into two thematic categories regarding medication 
adherence. Responses from patients were further classified according to those who had issues with medication-taking and those who did not have issues with medication-taking. Subthemes reflecting the reasons for issues included cost, effectiveness, inconvenience and side effects. Information regarding the patient's medication in addition to demographic data including patients' age, living arrangements, language background and presence of co-morbidities was also collected. Co-morbidities were further grouped into classes including neurological disorders, cardiovascular disorders and endocrine disorders such as diabetes mellitus. Furthermore, patients were classed as being English speaking or English as a second language (ESL). Additionally, information collected regarding patients' management of their medication-taking was classified into those who self-managed their medications or those who had a caregiver to assist this task. All collected data (interview, demographic and medical) were entered into the Statistical Package for the Social Sciences (SPSS, Version 19, Chicago) and the two thematic categories regarding adherence were compared with demographic data including patients' language background, co-morbidities and medication-taking management. Fisher's Exact Test was used to examine statistical associations between these factors. Moreover, data on the patients' current prescribed medications were compared with their responses provided within the thematic categories concerning adherence. 


\section{Results}

\section{Demographics, medications and complaints}

The average age of patients was $68 \pm 15.30$ years of age. The youngest patient was 40 years of age whereas the oldest patient was 90 years of age. As BPH is an age-dependent condition and because of the wide range of ages the patients were separated into two groups: younger and older. The younger group included patients under the age of 65 years whereas the older group included patients 65 years of age and over. There were seven (7) patients or $37 \%$ in the younger group and $12(63 \%)$ patients in the older group. Out of 12 patients in the older group, three (3) or 25\% were from an English speaking background, while the remaining nine (9) patients (75\%) were from an ESL background. In the younger group, three (3) patients (43\%) were from an English speaking background and four (4) patients (57\%) were from an ESL background.

In regards to living arrangements, no patients lived alone; all patients were either living with their spouse, parents, or children or within an aged care facility where nurses assisted with their medications. Two (2) patients in the younger group and nine (9) patients in the older group reported one or more co-morbidities. Co-morbidities reported in the younger group included renal failure, cognitive impairment, diabetes and coronary artery disease requiring double bypass whereas in the older group co-morbidities ranged from Alzheimer's disease, Parkinson's disease, hip replacement, stroke, thyroid problems, diabetes, hypertension and adrenal gland issues resulting in adrenalectomy. Patients with co-morbidities were on multiple medications, ranging from anti-Alzheimer's, antihypertensive, and antidiabetic agents however, majority of patients failed to identify them. Only two (2) patients in the older group had surgical procedure for their BPH known as transurethral resection of the prostate. No patients in the younger group had any type of surgery for their BPH. Out of 12 patients in the older group, six (6) patients or 50\% required the help of a caregiver to manage their 
medication-taking. By contrast, only one patient in the younger group required assistance with his medication.

A total of seven (7) different classes of pharmacological therapies including $5 \alpha$-RIs, $\alpha$ blockers, anticholinergics, plant extracts, antibiotics and combination of $\alpha$-blockers and $5 \alpha-$ RIs were prescribed. The top three medications prescribed to the older group were tamsulosin ( $\alpha$-blocker, 42\%), oxybutynin (anticholinergic, 33\%) and prazosin ( $\alpha$-blocker, $17 \%)$ while patients from the younger group were prescribed six different medications from the above mentioned classes of pharmacological therapies. The doses for tamsulosin, oxybutynin and prazosin were 400 micrograms mane, $5 \mathrm{mg}$ nocte and $0.5 \mathrm{mg}$ b.d. respectively. Only two (2) patients from the older group used a dosette box. In accordance to the thematic categories established based on the patients' response regarding issues with their current medications, answers were either classed as with or without issues. Issues with medication-taking were reported in five (5) older or $42 \%$ and six (6) younger or $86 \%$ patients (Table 1).

The use of the $\alpha$-blocker tamsulosin was reported by a total of six (6) patients, five (5) in the older and one in the younger group. Two (2) patients on tamsulosin, one from each group reported concerns relating to the medication's side effects and inconvenience. A total of five (5) patients used the anticholinergic medication, oxybutynin. Of these patients, three (3) had issues regarding the medication. The issues reported by two (2) older patients were medication effectiveness and inconvenience, while the single patient from the younger group raised an issue relating to the medication effectiveness only. Four (4) patients, two from each group were taking the $\alpha$-blocker, prazosin. Both patients from the younger group described issues relating to this medication. One was concerned about the medication side effects whereas another one reported inconvenience such as having to remember to take it every day. Single patient in the older group had concerns relating to medication's side effects. 


\section{Comparing demographics, medications and medication management}

Out of seven (7) patients in the younger group, six (6) patients reported issues regarding their current medications (Table 1). Two (2) patients reported reasons relating to inconvenience, three (3) patients reported reasons due to side effects, one patient reported concerns regarding the effectiveness of his current medication and one patient reported concerns relating to its cost. Only one patient under the age of 65 years required a caregiver to assist with the management of his medication-taking.

Out of 12 patients in the older group, five (5) or $42 \%$ reported issues concerning their current medications while the remaining seven (7) or 58\% did not report any issues (Table 1). Specifically, three (3) patients reported issues relating to inconvenience whereas two (2) patients reported reasons relating to side effects of the prescribed medication. Furthermore, one patient reported concerns regarding the effectiveness of his current medication. Additionally, six (6) of these 12 patients or $50 \%$ required their carers to manage their medication-taking.

Out of the six (6) patients who were from an English speaking background, four (4) or 67\% reported issues concerning their current medications. Their concerns ranged from costs of medications to side effects and inconvenience. No patients in this group reported any issues concerning effectiveness. In contrast, there were 13 patients from an ESL background and seven (7) or $54 \%$ reported issues concerning their current medications. Two (2) patients reported reasons relating to ineffectiveness whereas another two (2) patients reported reasons due to inconvenience. Furthermore, three (3) patients reported reasons concerning the side effect profile of their medication.

All eight (8) patients without any co-morbidities reported issues regarding their medications (Table 2). The most common complaint among patients without co-morbidities was side effects of a prescribed medication. In contrast, out of $11(58 \%)$ patients with co-morbidities, 
only three (3) had reported concerns relating to their medications (Table 2). Statistical analysis using the Fisher's Exact Test revealed that patients without co-morbidities were significantly more likely to have complaints with their medications compared to those with co-morbidities $(100 \%$ vs. $27 \%$, respectively $\mathrm{p}=0.002)$. Furthermore, seven (7) patients with co-morbidities required help of their caregivers to assist with medication-taking whereas only one patient without co-morbidities required a caregiver to manage his medication-taking. Statistical analysis showed that patients requiring the help of caregivers to manage their medication-taking were significantly less likely to have complaints with their medication compared to patients who self manage ( $29 \%$ vs. $75 \%$, respectively $\mathrm{p}=0.05)$ (Table 3 ). 


\section{Discussion}

The present study investigated the enablers and barriers affecting medication-taking behaviour in aging men with $\mathrm{BPH}$. We examined various parameters within the patients' demographics to help explain for differences in obstacles experienced by each demographic group regarding their medication-taking. Information was also obtained on enablers for medication-taking.

This study found that patients younger than 65 years of age were more likely to have issues regarding their current medications than those who were older than 65. This information helps explain the reasons for poor adherence in younger patients with $\mathrm{BPH}$ as described in previous studies $[17,18]$. Older adults, female gender, and consumers' belief that therapy will help are additional predictors of adherence [20]. Furthermore, we also found that younger patients were less likely to require caregivers' assistance with the management of their medications when compared to those in the older age group which was to be expected. Yet patients who self-manage had greater concerns regarding their medications than those who required the aid of carers to manage their medication-taking. A lack of belief in the effectiveness of the prescribed medication and a lack of support have been associated with weak medication adherence. With the aid of carers, patients miss their medications less frequently, receive better social or family support and are better informed about their medications [25-28]. Thus, the findings of our study support better adherence seen in older BPH patients described in previous studies $[17,18]$.

The present study also found that patients from an English speaking background were more likely to report complaints with their medications when compared to those from a linguistically diverse background. The reason for this can be explained by a better language concordance with their physician, thus better health literacy regarding health outcomes and

medication knowledge [29, 30]. Conversely, this study found that patients from a 
linguistically diverse background were less likely to report issues when compared to those from an English speaking background, suggesting good adherence. It is likely that patients from a linguistically diverse background will have poorer health literacy regarding their medication knowledge $[29,30]$. This may be due to the likelihood of language barriers and miscommunication with their healthcare providers, [31] and not being able to articulate their concerns. Several studies have described poor health literacy to be associated with poor adherence to medications [29, 32, 33]. Having a language barrier will most likely prevent patients with ESL backgrounds to improve their literacy skills [31]. Yet, our study supports the opposite, suggesting fewer complaints with medication-taking from those from a linguistically diverse background when compared to those from an English speaking background. A key possible explanation may be linked to the cultural aspects among these patients. Those from culturally diverse backgrounds tend to value family closeness and loyalty, therefore having family support helps them to better adhere to pharmacological therapy [27].

Patients without co-morbidities had significant issues regarding their medications compared to those with co-morbidities, suggesting problems with adherence. The most common reason for complaint was related to the medications' side effects and inconvenience in medicationtaking. A possible explanation for the high incidence of reported side effects may be due to the likelihood that these patients only experience symptoms exclusively associated with their $\mathrm{BPH}$ condition. It is known that patients experiencing other significant bothersome concerns will tend to complain less about minor side effects of medications compared to patients with minimal and less bothersome symptoms [34, 35]. Furthermore, reported inconvenience in medication-taking among these patients can be possibly due to not being used to taking medications long-term and a lack of dependency on carers for the management of their medications, therefore limiting the support from others to take their prescribed medications 
[25]. In contrast, patients with co-morbidities were considerably less likely to describe having issues with their current medications compared to patients without co-morbidities. This may suggest medication concerns are minor when compared to the various symptoms of comorbidities and side effects of multiple medications used to treat these co-morbidities. In addition, better adherence in this group may be possible, contradicting many other studies suggesting co-morbidities being associated with increased issues with compliance and poor adherence [36, 37]. This is likely the result of greater dependency on carers for the management of medication-taking among patients with co-morbidities. Another possible explanation for the lack of reported issues among individuals with co-morbidities can be described by the Health Belief Model, where severely ill patients tend to take medications as prescribed due to the perceived necessity to treat their condition effectively [38].

In conclusion, the findings of the present study suggest that demographic data relating to the patients' age, language background, co-morbidities and medication-taking management style can affect medication adherence in men with BPH. Younger patients were more likely to have complaints regarding their medication compared with older patients, which may suggest weaker adherence. Moreover, older patients were more likely to receive the assistance of caregivers in the management of their medication-taking, suggesting better adherence. Furthermore, those from a linguistically diverse background were less likely to report having issues with their medications despite a likelihood of having a language barrier compared to those from an English speaking background. Thus, cultural factors may play an important role in medication adherence. In addition, those receiving aid from their carers for medicationtaking were considerably less likely to have complaints regarding their medications as opposed to those not requiring the assistance of caregivers. This highlights the importance of having support for medication-taking in these patients to assist with better adherence. Additionally, issues concerning cost, effectiveness, inconvenience and side effects were 
identified as major barriers affecting medication-taking. Subsidisation occurs with many prescribed medications in Australia through the Pharmaceutical Benefits Scheme (PBS), which is an initiative of the Federal Government. The aim of this subsidisation is to help Australian citizens to access necessary and cost-effective therapies. Further subsidisations are available for individuals receiving concessional benefits, such as older people and the unemployed. However, despite the availability of the PBS, medication costs can still act as a barrier for consumers, as indicated in past work [39]. Two limitations of this study are small sample size and lack of detail in relation to medications prescribed to patients with comorbidities. Future investigations should focus on detailed exploration of other medications used by patients with BPH in a larger sample size to help draw better inferences concerning medication complaints. Furthermore, assessing the complexity of medication regimens prescribed for other conditions can help address the ambiguity concerning the reported complaints in the present study. Finally, assessing the frequency of missing a dose of BPH medication and clarifying the reasons behind patients' decision to miss a dose could provide further information necessary to develop strategies to enhance medication-taking in this group. 


\section{Acknowledgements}

We would like to thank the staff at the Melbourne Health LUTS clinic for helping in recruitment and the patients who agreed to be interviewed for this study. 


\section{Declaration of interest}

The authors report no conflicts of interest. 


\section{Appendix 1}

Structured interview questionnaire

1. Can you tell me a bit about yourself? Where do you live / living arrangements / age / completed years of schooling?

2. Main language spoken at home.

3. What do you know about your medical condition? Can you explain it in few words?

4. What medications are you taking for your condition?

5. For how long have you been taking the prescribed medications?

6. Can you tell me what helps you take your medications?

7. What don't you like about taking your medications?

8. What do you like about taking your medications?

9. What is the reason for seeing the urologist today?

10. Do you have any other concerns that you wish to point out? 


\section{References}

1. Matyus P,Horvath K. Alpha-adrenergic approach in the medical management of benign prostatic hyperplasia. Med Res Rev 1997; 17:523-535.

2. Pennefather JN, Lau WA, Mitchelson F, Ventura S. The autonomic and sensory innervation of the smooth muscle of the prostate gland: a review of pharmacological and histological studies. J Auton Pharmacol 2000; 20:193-206.

3. Roehrborn C. Benign prostatic hyperplasia and lower urinary tract symptom guidelines. Can Urol Assoc J 2012; 6:S130-132.

4. Thorpe A,Neal D. Benign prostatic hyperplasia. Lancet 2003; 361:1359-1367.

5. Roehrborn CG. Pathology of benign prostatic hyperplasia. Int J Impot Res 2008; 20 Suppl 3:S11-S18.

6. Taub DA,Wei JT. The economics of benign prostatic hyperplasia and lower urinary tract symptoms in the United States. Curr Urol Rep 2006; 7:272-281.

7. Jacobsen SJ, et al. Treatment for benign prostatic hyperplasia among community dwelling men: the Olmsted County study of urinary symptoms and health status. J Urol 1999; 162:1301-1306.

8. Miner MM. Primary care physician versus urologist: how does their medical management of LUTS associated with BPH differ? Curr Urol Rep 2009; 10:254-260.

9. de la Rosette JJ, et al. EAU Guidelines on benign prostatic hyperplasia (BPH). Eur Urol 2001; 40:256-263; discussion 264.

10. Madersbacher S, Alivizatos G, Nordling J, Sanz CR, Emberton M,de la Rosette JJ. EAU 2004 guidelines on assessment, therapy and follow-up of men with lower urinary tract symptoms suggestive of benign prostatic obstruction (BPH guidelines). Eur Urol 2004; 46:547-554.

11. Reynard JM. Does anticholinergic medication have a role for men with lower urinary tract symptoms/benign prostatic hyperplasia either alone or in combination with other agents? Curr Opin Urol 2004; 14:13-16.

12. Lam JS, Romas NA,Lowe FC. Long-term treatment with finasteride in men with symptomatic benign prostatic hyperplasia: 10-year follow-up. Urology 2003; 61:354358.

13. Cervenakov I, Fillo J, Mardiak J, Kopecny M, Smirala J,Lepies P. Speedy elimination of ureterolithiasis in lower part of ureters with the alpha 1-blocker--Tamsulosin. Int Urol Nephrol 2002; 34:25-29. 
14. Chapple CR. Alpha adrenoceptor antagonists in the year 2000: is there anything new? Curr Opin Urol 2001; 11:9-16.

15. Gleason DM, Susset J, White C, Munoz DR,Sand PK. Evaluation of a new once-daily formulation of oxbutynin for the treatment of urinary urge incontinence. Ditropan XL Study Group. Urology 1999; 54:420-423.

16. Leedahl DD, Vo PH, Maxson PM,Lovely JK. Benign Prostatic Hyperplasia: Implications for Pharmacologic Treatment and Perioperative Care. J Pharm Pract 2012;

17. Lin YH, Jiang YG, Wang JS,Luo Y. Finasteride adherence-associated factors in Chinese benign prostatic hyperplasia patients. Urol Int 2012; 88:177-182.

18. Nichol MB, Knight TK, Wu J, Barron R,Penson DF. Evaluating use patterns of and adherence to medications for benign prostatic hyperplasia. J Urol 2009; 181:22142221; discussion 2221-2222.

19. Yu YF, Nichol MB, Yu AP,Ahn J. Persistence and adherence of medications for chronic overactive bladder/urinary incontinence in the california medicaid program. Value Health 2005; 8:495-505.

20. Haynes RB, Ackloo E, Sahota N, McDonald HP,Yao X. Interventions for enhancing medication adherence. Cochrane Database Syst Rev 2008; CD000011.

21. Johnson MJ. The Medication Adherence Model: a guide for assessing medication taking. Res Theory Nurs Pract 2002; 16:179-192.

22. Pound P, et al. Resisting medicines: a synthesis of qualitative studies of medicine taking. Soc Sci Med 2005; 61:133-155.

23. Lawton J, Peel E, Parry O,Douglas M. Patients' perceptions and experiences of taking oral glucose-lowering agents: a longitudinal qualitative study. Diabet Med 2008; 25:491-495.

24. Williams A, Manias E,Walker R. Interventions to improve medication adherence in people with multiple chronic conditions: a systematic review. J Adv Nurs 2008; 63:132-143.

25. Ammassari A, et al. Correlates and predictors of adherence to highly active antiretroviral therapy: overview of published literature. J Acquir Immune Defic Syndr 2002; 31 Suppl 3:S123-127.

26. Barclay TR, et al. Age-associated predictors of medication adherence in HIV-positive adults: health beliefs, self-efficacy, and neurocognitive status. Health Psychol 2007; 26:40-49. 
27. Burge S, et al. Correlates of medication knowledge and adherence: findings from the residency research network of South Texas. Fam Med 2005; 37:712-718.

28. Cohen I, Rogers P, Burke V,Beilin LJ. Predictors of medication use, compliance and symptoms of hypotension in a community-based sample of elderly men and women. $\mathbf{J}$ Clin Pharm Ther 1998; 23:423-432.

29. Kalichman SC,Rompa D. Functional health literacy is associated with health status and health-related knowledge in people living with HIV-AIDS. J Acquir Immune Defic Syndr 2000; 25:337-344.

30. Traylor AH, Schmittdiel JA, Uratsu CS, Mangione CM,Subramanian U. Adherence to cardiovascular disease medications: does patient-provider race/ethnicity and language concordance matter? J Gen Intern Med 2010; 25:1172-1177.

31. Cooper LA, Roter DL, Johnson RL, Ford DE, Steinwachs DM,Powe NR. Patientcentered communication, ratings of care, and concordance of patient and physician race. Ann Intern Med 2003; 139:907-915.

32. Gazmararian JA, Kripalani S, Miller MJ, Echt KV, Ren J,Rask K. Factors associated with medication refill adherence in cardiovascular-related diseases: a focus on health literacy. J Gen Intern Med 2006; 21:1215-1221.

33. Nichols-English G,Poirier S. Optimizing adherence to pharmaceutical care plans. J Am Pharm Assoc (Wash) 2000; 40:475-485.

34. Michel MC, Mehlburger L, Schumacher H, Bressel HU,Goepel M. Effect of diabetes on lower urinary tract symptoms in patients with benign prostatic hyperplasia. J Urol 2000; 163:1725-1729.

35. Speakman MJ. Lower urinary tract symptoms suggestive of benign prostatic obstruction: what is the available evidence for rational management? Eur Urol 2001; 39 Suppl 3:6-12.

36. Monane M, Bohn RL, Gurwitz JH, Glynn RJ, Levin R,Avorn J. The effects of initial drug choice and comorbidity on antihypertensive therapy compliance: results from a population-based study in the elderly. Am J Hypertens 1997; 10:697-704.

37. Nikolaus T, Kruse W, Bach M, Specht-Leible N, Oster P,Schlierf G. Elderly patients' problems with medication. An in-hospital and follow-up study. Eur J Clin Pharmacol 1996; 49:255-259.

38. Janz NK,Becker MH. The Health Belief Model: a decade later. Health Educ Q 1984; 11:1-47. 
39. Manias E, Claydon-Platt K, McColl GJ, Bucknall TK,Brand CA. Managing complex medication regimens: perspectives of consumers with osteoarthritis and healthcare professionals. Ann Pharmacother 2007; 41:764-771. 
Table 1 Frequency of reported complaints and associated reasons between age groups

\begin{tabular}{ccccccc}
\hline \multirow{2}{*}{$\begin{array}{c}\text { Age } \\
\text { group } \\
\text { (years) }\end{array}$} & Total & $\%$ & \multicolumn{5}{c}{ Reasons } \\
\cline { 5 - 7 } & $(\mathrm{n})$ & Complaints & Cost & Effectiveness & Inconvenience & Side effect \\
\hline$<65$ & 7 & 86 & 1 & 1 & 2 & 3 \\
$\geq 65$ & 12 & 42 & 0 & 1 & 3 & 2 \\
\hline
\end{tabular}


Table 2 Frequency of reported complaints and associated reasons

between co-morbidity statuses

\begin{tabular}{|c|c|c|c|c|c|c|}
\hline \multirow{2}{*}{ Co-morbidities } & \multirow{2}{*}{$\begin{array}{l}\text { Total } \\
\text { (n) }\end{array}$} & \multirow{2}{*}{$\begin{array}{c}\% \\
\text { Complaints }\end{array}$} & \multicolumn{4}{|c|}{ Reasons } \\
\hline & & & Cost & Effectiveness & Inconvenience & Side effect \\
\hline Without & 8 & 100 & 1 & 1 & 3 & 5 \\
\hline With & 11 & $27 * *$ & 0 & 1 & 2 & 0 \\
\hline
\end{tabular}

$* * \mathbf{p}=\mathbf{0 . 0 0 2}$ 
Table 3 Frequency of reported complaints and associated reasons

between medication-taking management styles

\begin{tabular}{ccccccc}
\hline \multirow{2}{*}{$\begin{array}{c}\text { Medication } \\
\text { management }\end{array}$} & $\begin{array}{c}\text { Total } \\
(\mathrm{n})\end{array}$ & $\begin{array}{c}\% \\
\text { Complaints }\end{array}$ & Cost & Effectiveness & Inconvenience & $\begin{array}{c}\text { Side } \\
\text { effect }\end{array}$ \\
\hline \hline Caregiver & 7 & 29 & 0 & 0 & 2 & 0 \\
Self & 12 & $75^{* *}$ & 1 & 2 & 3 & 5 \\
\hline
\end{tabular}

$* \mathbf{p}=0.05$ 